Brazilian Journal

of Chemical

ISSN 0104-6632

Engineering

\title{
PREDICTION OF EFFECTIVE DIFFUSIVITY TENSORS FOR BULK DIFFUSION WITH CHEMICAL REACTIONS IN POROUS MEDIA
}

\author{
E. A. Borges da Silva, D. P. Souza, A. A. Ulson de Souza, \\ and S. M. A. Guelli U. de Souza* \\ Departamento de Engenharia Química e de Engenharia de Alimentos, Universidade Federal de Santa Catarina, \\ Laboratório de Simulação Numérica de Sistemas Químicos, LABSIN, Phone: (+55) (48) 3331-9448, \\ Fax: (+55) (48) 3331-9687, Campus Universitário, Cx. P. 476, CEP: 88.040-900, Florianópolis - SC, Brazil \\ E-mail: eduardo@fe.up.pt,E-mail: selene@enq.ufsc.br
}

(Received: June 27, 2005 ; Accepted: August 30, 2006)

\begin{abstract}
In this paper, the volume averaging transport equations of two reactive processes in porous media are presented. The porous media are characterized by different length scales and the information describing the mass transfer mechanisms is transferred hierarchically between scales by applying the volume averaging method. This development provides the theoretical definition of effective transport coefficients, which can be predicted through solution of the closure problems. The theoretical calculation of effective diffusion tensors of the species in the particle pores is presented. Two closure problems are deduced through mathematical formulation of two different scales: microporous (process 1) and macroporous (process 2). In order to solve these closure problems, the volume finite method is used as the numerical methodology. Good agreement is verified between the numerical solutions obtained in this study and the data found in the literature for the closure problems considered.

Keywords: Effective diffusivity tensor; Method of volume averaging; Closure problems; Porous media.
\end{abstract}

\section{INTRODUCTION}

In reaction engineering, the separation and reaction processes involve different transfer phenomena: adsorption, diffusion/dispersion and convection, besides the chemical/biochemical reaction, which can be homogeneous and/or heterogeneous. If these phenomena can be accurately described through the development of mathematical formulations, such formulations will be useful in the design and optimization of processes in any operating units.

In some reactors where chemical reactions take place in the presence of a stationary phase, there is usually a heterogeneous porous medium, which is composed of many scales. Some heterogeneity levels found in a chromatographic reactor are schematized in Fig. 1. This heterogeneity is characterized by length scales that include from the largest length scale, related to the reactive unit, to the smallest length scale, related to the adsorption and/or reaction sites of the particles.

*To whom correspondence should be addressed 


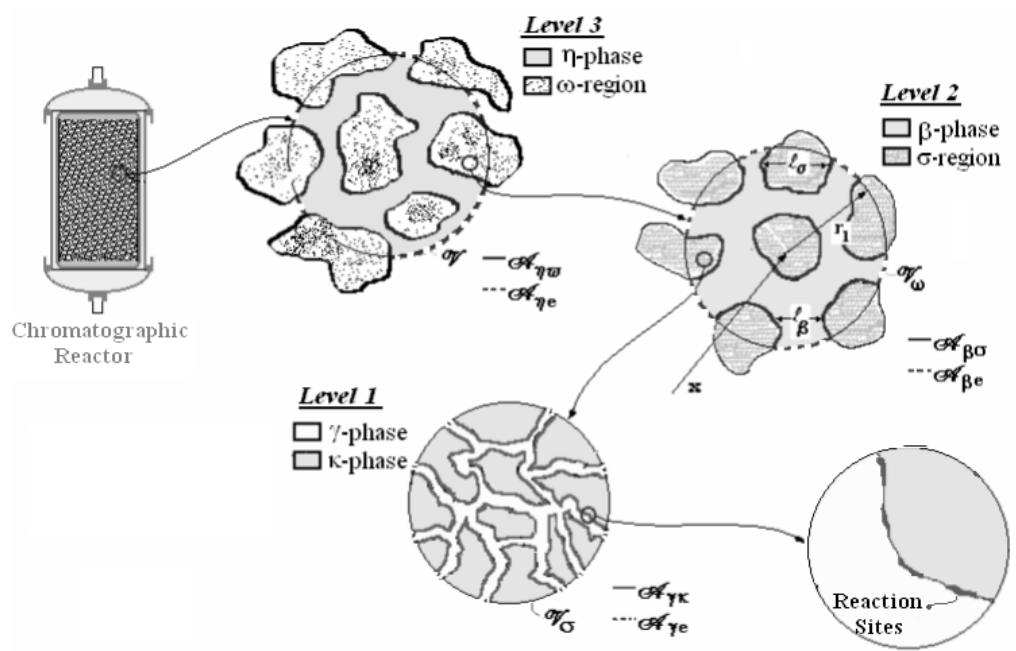

Figure 1: Hierarchical structure of length scales in a porous medium of a chromatographic reactor.

When significantly different length scales are involved in a hierarchical system, the method of volume averaging can be used to take the information from the particle scale of the reactive multiphase flow, comprising the pores and the solid itself, to the macroscopic scale of measurable amounts (Nguyen et al., 1982; Wood and Whitaker, 1998). This development provides the theoretical definition of effective transport coefficients, which can be predicted by solving some boundary value problems, called closure problems (Crapiste et al., 1986). The method of volume averaging has been applied successfully in dealing with some problems of transport phenomena in porous media: active and passive dispersion (Quintard and Whitaker, 1994), dispersion with chemical reaction (Ryan et al., 1981; Guelli U. de Souza and Whitaker, 2003), and dispersion with adsorption (Plumb and Whitaker, 1990; Ulson de Souza and Whitaker, 2003), among others.

Since the diffusion process in porous media is a subject of great interest in engineering, mainly in the design of chemical/biochemical reactors, the solution of two closure problems derived from the application of volume averaging in the governing differential equations of biotechnological problems is presented. The first closure problem refers to the microscale of a packed bed reactor in the glucose isomerization process and the second is associated with the intermediate scale of a chromatographic reactor in the sucrose inversion process. The aim is to show the potential of this approach, in which the effective diffusivity in porous particles with different length scales can be theoretically calculated from the solution of closure problems. The closure problems are solved by the numerical techniques of finite volumes and generalized coordinate systems. Numerical solutions are performed using spatially periodic models of porous media.

\section{DESCRIPTION OF THE PROCESS - MICROSCALE CASE}

The first process to be considered is convectivediffusive transport in the presence of a reversible chemical reaction, in which species $A$ is consumed to produce species $B$ (for example, the glucose isomerization process, glucose $\leftrightarrows$ fructose). The reaction takes place in the pores of the catalyst pellets - glucose isomerase immobilized enzyme in a packed bed reactor. The resultant mixture of the species must be selectively separated through a subsequent separation process. The hierarchical structure of the porous medium under consideration in this formulation is illustrated in Fig. 2.

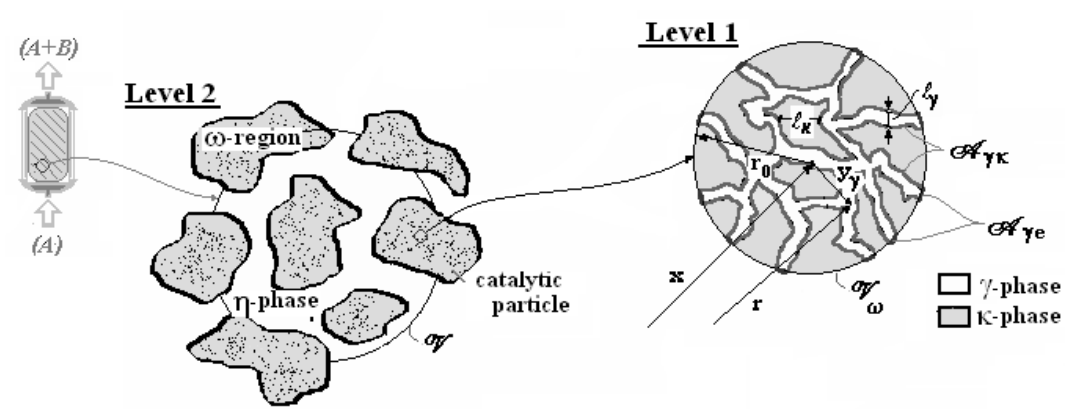

Figure 2: Hierarchical structure of the catalytic porous medium in a packed bed reactor. 
On the macroscale or design scale (level 2 in Fig. 2 ), there is a fluid region, identified as the $\eta$-region, which surrounds the solid particles packed in the reactor - $\omega$-region. Level 1 corresponds to the microscale and represents the porous region of the particles. In the averaging volume of the microscale $\left(\mathscr{V}_{\omega}\right)$, the $\kappa$-region is the solid matrix of the catalyst and the fluid phase within the pores of the particle is identified as the $\gamma$-phase. The $\gamma$ - $\kappa$ interface is a catalytic surface. In Fig. 2, the position vector $\boldsymbol{r}$ is used to localize any point in three-dimensional space. The position vector $\mathbf{x}$ determines the centroid of the averaging volume and the position vector $\mathbf{y}_{\gamma}$ is used to represent points in the $\gamma$-phase relative to the centroid of $\mathscr{Q}_{\omega}$.

For the microscale, the reactive system under consideration $(A \leftrightarrows B)$ is described by the following governing transport equations and interface boundary conditions:

$\frac{\partial C_{i \gamma}}{\partial t}=\nabla \cdot\left(\mathscr{D}_{i \gamma} \nabla C_{i \gamma}\right), \quad$ in $\gamma$-phase

B.C.1

$$
+\mathbf{n}_{\gamma \kappa} \cdot \mathscr{D}_{i \gamma} \nabla C_{i \gamma}=\delta_{i} R_{i}, \quad \text { in } \mathscr{A}_{\gamma \kappa}
$$

\section{B.C.2}

$$
C_{i \gamma}=\mathscr{F}_{i}(\mathbf{r}, t), \quad \text { in } \mathscr{A}_{\gamma e}
$$

I.C.

$C_{i \gamma}=\mathscr{G}_{i}(\mathbf{r}), \quad$ in $t=0$

In Eqs.(1)-(4), $C_{i \gamma}$ is the punctual concentration in the $\gamma$-phase of averaging volume $\mathscr{V}_{\omega}$ of species $i$, $\mathscr{A}_{\gamma \kappa}$ is the $\gamma-\kappa$ interfacial area, and $\mathscr{D}_{i}$ is the molecular diffusivity of species $i$. The reaction rate term is given by $R_{i}\left(\mathrm{~mol} / \mathrm{s} \mathrm{m}^{2}\right)$ and the variable $\delta$ can have values of 1 and -1 for the reagent species and product, respectively.

The boundary condition given by Eq.(3) is basically used to show that the information concerning the punctual concentration in $\mathscr{A}_{\gamma e}$ (area of the $\gamma$-phase entrances and exists associated with the microscopic region in Fig. 2) is not known. The same applies to the initial condition. For the glucose isomerization process, the global reaction rate may be based on the reversible Briggs-Haldane mechanism.

The spatially smoothed form of Eq.(1) is given by

$$
\begin{aligned}
& \varepsilon_{\gamma} \frac{\partial\left\langle\mathrm{C}_{\mathrm{i} \gamma}\right\rangle^{\gamma}}{\partial \mathrm{t}}= \\
& \nabla .\left[\mathscr{D}_{\mathrm{i} \gamma}\left(\varepsilon_{\gamma} \nabla\left\langle\mathrm{C}_{\mathrm{i} \gamma}\right\rangle^{\gamma}+\frac{1}{\mathscr{W}_{\omega}} \int_{\mathrm{A}_{\gamma \kappa}} \mathrm{n}_{\gamma \kappa} \tilde{\mathrm{C}}_{\mathrm{i}} \mathrm{dA}\right)\right]+ \\
& +\left.\delta_{\mathrm{i}} \mathrm{a}_{\mathrm{v}}\right|_{\gamma \kappa}\left\langle\mathrm{R}_{\mathrm{i}}\right\rangle_{\gamma \kappa}
\end{aligned}
$$

in which the preferred concentration for describing the mass transfer process in porous media is the intrinsic average concentration defined as

$$
\left\langle C_{i_{n}}\right\rangle^{n}=\frac{1}{V_{n}} \int_{V_{n}} C_{i_{n}} d V
$$

for a given $n$-phase or $n$-region ( $V_{n}, n$-phase volume of the averaging volume). The volume fraction of the $n$-phase (or $n$-region) is defined explicitly by

$$
\varepsilon_{n}=\frac{V_{n}}{\mathscr{N}_{m}}
$$

( $m$ is the macroregion that contains the $n$-phase or $n$ region)

In order to derive Eq.(5) from Eqs.(1)-(4), we have made some simplifications, all of which are associated with the two length scale constraints,

$$
\ell_{\gamma}<<r_{o} ; \quad r_{o}<<L
$$

In Eq.(8), $\ell_{\gamma}$ is the length scale for the $\gamma$-phase, $r_{o}$ is the radius of averaging volume $\mathscr{T}_{\omega}$ and $L$ is the macroscopic length scale. Borges da Silva (2004) presented the smoothing procedure for the reversible reaction under study. To obtain Eq.(5), we used the spatial deviation concentration $(\widetilde{C})$ according to

$$
\widetilde{C}_{i n}=C_{i n}-\left\langle C_{i n}\right\rangle^{n}
$$




\section{Closure}

In order to develop a closed form of Eq.(5), one should obtain representations for the spatial deviation concentrations. This can be achieved by subtraction of the punctual equations for the respective volume-averaged equations. This procedure should be considered in the development of the closure problem. The following representation for $\widetilde{C}_{i \gamma}$ is proposed:

$$
\widetilde{C}_{i \gamma}=\left.\mathrm{b}_{i \gamma} \cdot \nabla\left\langle C_{i \gamma}\right\rangle^{\gamma}\right|_{\mathrm{x}}+\left.s_{i \gamma}\left\langle R_{i}\right\rangle_{\gamma \kappa}\right|_{\mathrm{x}}+\psi_{i \gamma}
$$

The vector $\boldsymbol{b}_{i \gamma}$ and the scalar $s_{i \gamma}$ are referenced as closure variables and $\psi_{i \gamma}$ is an arbitrary function. The closure variables can be determined through the boundary value problems, as exemplified by field $\boldsymbol{b}_{\boldsymbol{i} \gamma}$ :

$$
\nabla^{2} \mathbf{b}_{i \gamma}=0
$$

B.C.1

$$
-\mathbf{n}_{\gamma \kappa} \cdot \nabla \mathbf{b}_{i \gamma}=\mathbf{n}_{\gamma \kappa}, \quad \text { in } \mathscr{A}_{\gamma \kappa}
$$

Periodicity:

$\mathbf{b}_{i \gamma}\left(\mathbf{r}+\ell_{j}\right)=\mathbf{b}_{i \gamma}(\mathbf{r}) \quad j=1,2,3$

In closure problems, $\ell_{j}$ represents the three lattice vectors that describe a spatially periodic media (Brenner, 1980). Since for resolution of the concentration deviation field a spatially periodic model of the porous medium is used, the contribution of $\psi_{i \gamma}$ to the closed form of the governing equation for the microscale is null. It is possible to show that there is no significant contribution of the second term of Eq.(10), the term of the scalar $s_{i \gamma}$, to the field of $\left\langle C_{i \gamma}\right\rangle^{\gamma}$ in relation to its respective source term (Ryan et al., 1981).

The governing equation for the microscale, Eq.(14), which describes the species transfer from the fluid phase to the catalyst porous surface, is obtained through use of representation for $\widetilde{C}_{i \gamma}$ in the intrinsic concentration transport equation. Thus, the closed form of this volume averaged mass conservation equation is expressed as

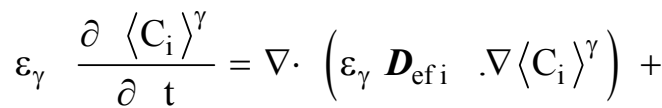

$$
\begin{aligned}
& +\left.\delta_{\mathrm{i}} \quad \mathrm{a}_{\mathrm{v}}\right|_{\gamma \kappa}\left\langle\mathrm{R}_{\mathrm{i}}\right\rangle_{\gamma \kappa}
\end{aligned}
$$

with the effective diffusivity tensor of species $i \boldsymbol{D}_{\text {efi }}$ defined by

$$
\left.\boldsymbol{D}_{\text {ef } i}\right|_{\gamma \kappa}=\mathscr{D}_{i \gamma}\left(\mathbf{I}+\frac{1}{V_{\gamma}} \int_{A_{\gamma \kappa}} \mathbf{n}_{\gamma \kappa} \mathbf{b}_{i \gamma} d A\right)
$$

\section{Microscale Closure Problem}

For the microscale of the reactor packed with catalyst, the solution of the closure variable field $\boldsymbol{b}_{\boldsymbol{i} \gamma}$ specified by Eqs.(11)-(13) allows calculation of the effective diffusivity tensor of catalytic particles using Eq.(15).

In Eq.(15), one can observe that there are two terms controlling the value of the effective diffusivity tensor: the term $\mathscr{D}_{i \gamma} \mathbf{I}$ (only molecular diffusivity) and a second term that consists of a geometric tensor $\left(\boldsymbol{b}_{\boldsymbol{i}}\right)$ multiplied by the molecular diffusivity. Since effective diffusivity is independent of reaction rate (Crapiste et al., 1986), we can conclude that it is sufficient to consider the passive dispersion case in this porous medium.

The closure problem for $\boldsymbol{b}_{\boldsymbol{i} \gamma}$ is solved for two models of spatially periodic porous media: the twodimensional array of cylinders (Fig. 3a) and the twodimensional array of squares (Fig. 3b). The former was originally solved by Ryan et al. (1981) and the latter by Ochoa-Tapia et al. (1994). The unit cells can be defined with a high degree of complexity, although the predicted theoretical values for the effective coefficients will be very close to those found using periodic models, such as those illustrated in Fig. 3. This is related to the characteristics of the boundary value problem for the filter represented by the area integral in Eq.(15) (Quintard and Whitaker, 1993).

Both arrays in Fig. 3 are isotropic with respect to the diffusion process in the $x-y$ plane; therefore, it is only necessary to determine a single component $\boldsymbol{b}_{\boldsymbol{i} \gamma}$ of $\left.\boldsymbol{D}_{\mathrm{ef} i}\right|_{\gamma \kappa}$. This is not the case for porous media, which are neither isotropic nor homogeneous. A discussion of systems where there is some level of anisotropy with respect to the diffusion can be found in Whitaker (1999). 


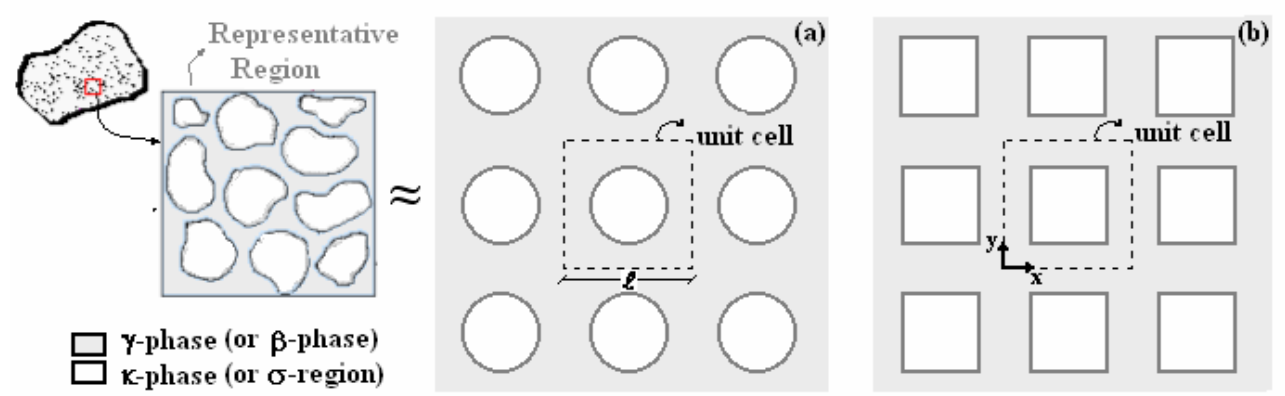

Figure 3: Spatially periodic models of porous media: (a) two-dimensional array of cylinders; (b) two-dimensional array of squares.

The differential equations are discretized by the finite volume method and the fictitious volumes technique is used for application of the boundary conditions. The equations are written in a general curvilinear coordinate systems allowing the numerical procedure to be carried out in a transformed plane (Maliska, 1995). The algebraic equations are implemented in a computational algorithm using FORTRAN language. The convergence of the solution is evaluated by increasing the number of control volumes until no change is observed between different runs.

The two-dimensional field of $\boldsymbol{b}$ is compared to that shown in Ochoa-Tapia et al. (1994) for cylindrical arrays with porosity $\varepsilon_{\gamma}$ equal to 0.84 . In Fig. 4, the values of $b_{x}$ appear in a dimensionless way through the lattice vectors. Reasonable agreement between the isolines of the $b_{x}$ field obtained in this study and those from the literature can be observed. The numerical method used by Ochoa-Tapia et al. is a finite-difference scheme. It is worth mentioning that the effective diffusivity in Eq.(15) is determined by the value of $b_{x}$ at the $\gamma-\kappa$ interface and this value is given by the governing differential equation and the flux boundary condition (only weakly influenced by the periodicity condition). In this case, the numerical results from our study are in good agreement with those published in the literature.

The analytical solution of the closure problem given by Eqs.(11)-(13) is possible if Chang's unit cell is adopted and the periodicity condition of Eq.(13) can be replaced by a Dirichlet condition specifying $\boldsymbol{b}=0$ at the external limit of the unit cell (i.e., $r=r_{2}$ in Fig. 5)(Chang, 1983). Figure 5 shows the numerical results for the effective diffusivity when the cylindrical model of Chang's unit cell is applied. The numerical methodology is the same as that used in the solution of the microscale closure problem for the unit cell shown in Fig. 3. The numerical results are compared with the analytical solution presented by Quintard and Whitaker (1993).

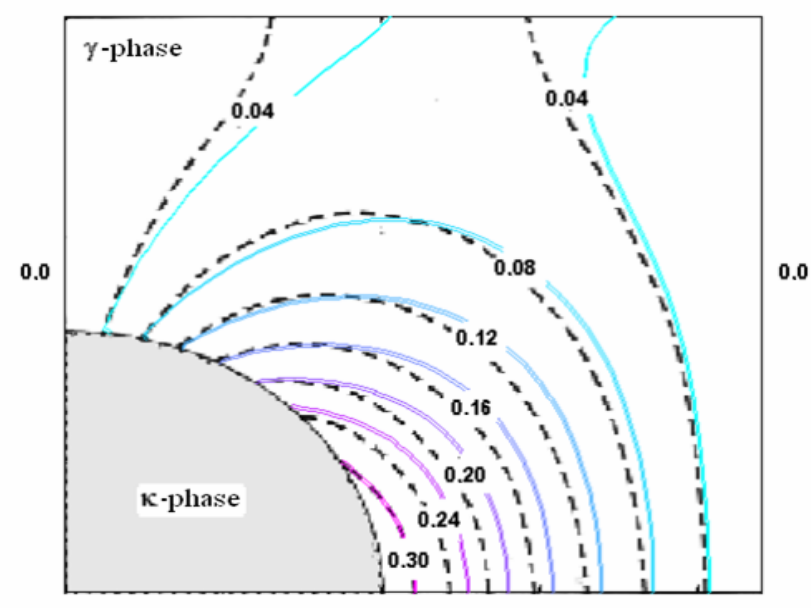

Figure 4: Field of $2 b_{x} / \ell$ calculated for the porous medium with cylindrical array.

(-) this study, (- - ) Ochoa-Tapia et al. (1994). 


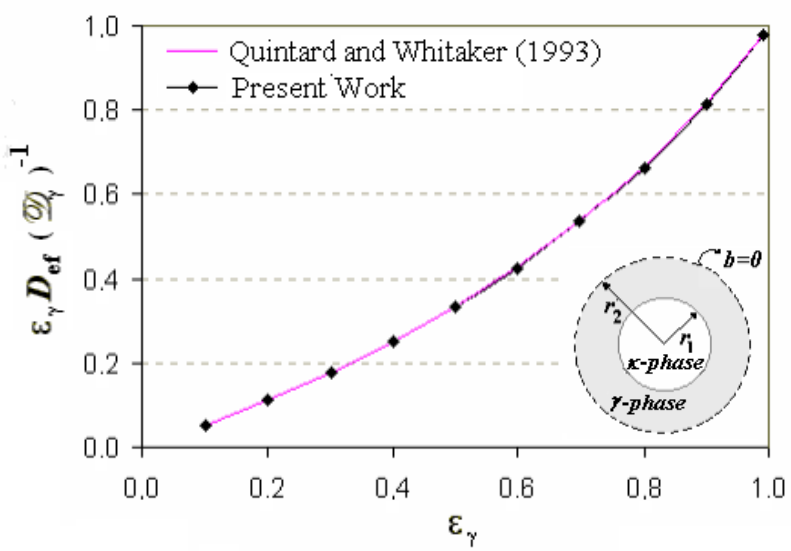

Figure 5: Solution of the microscale closure problem using Chang's unit cell.

One can verify good agreement between the results obtained in this study and those obtained by Quintard and Whitaker (1993), validating the numerical methodology. Ochoa- Tapia et al. (1994) presented a brief analysis of Chang's unit cell for the calculation of $\left.\boldsymbol{D}_{\text {ef } i}\right|_{\gamma \kappa}$.

The values for the isotropic effective diffusivity tensor, calculated using the spatially periodic models of porous media illustrated in Fig. 3, are shown in Fig. 6. Here one can also observe the numerical results obtained by Ryan et al. (1981) and by Quintard and Whitaker (1993), in which a three-dimensional model of spheres, along with some experimental data, was used (Whitaker, 1999).

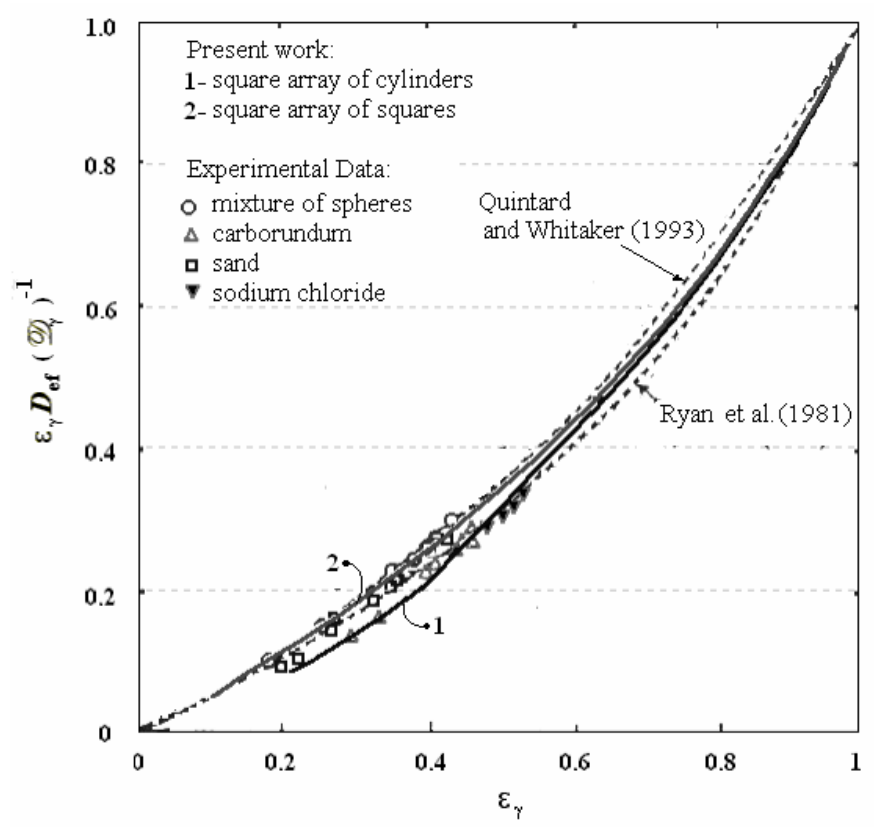

Figure 6: Dimensionless effective diffusivity for nonconsolidated pores.

Good agreement between the numerical curves found in this study and those of other researchers can be verified in Fig. 6. There is also good agreement between the theoretical model and the experimental data. It is important to note that the experimental data are obtained from disordered systems, i.e., from spatially nonperiodic systems.
For the glucose isomerization process where the glucose isomerase enzyme, which can be immobilized on several types of supports, is used, the effective diffusivity values for glucose diffusion are in the range of $10^{-9}$ to $10^{-6} \mathrm{~cm}^{2} / \mathrm{s}$ (Linko et al., 1981). Asif and Abasaeed (1998) declared that there is a lack of reliable knowledge concerning glucose 
diffusivity in biocatalysts. Such effective diffusivity has been described as a function of particle porosity $\left(\varepsilon_{p}\right)$ and tortuosity (Linko et al., 1981). Generally, the porosity values are between 0.4 and 0.8 and the tortuosity values are between 1 and 6 . The value ranges for these parameters provide an effective diffusivity between $0.1 \mathscr{D}$ and $0.8 \mathscr{D}$. Table 1 shows the effective diffusivity values and other correlations, which have been reported in the literature for glucose diffusion in some particle types. In Table 2 , some molecular diffusivity data for glucose at $25^{\circ} \mathrm{C}$ and in different concentrations are presented.

Table 1: Experimental effective diffusivities and some correlations for glucose diffusion in porous media.

\begin{tabular}{|l|c|c|}
\hline References & $\varepsilon_{p}$ & Effective Diffusivity $\left(\mathbf{c m}^{2} / \mathbf{s}\right)$ \\
\hline Linko et al. (1981) & 0.8 & $2.610^{-6^{*}}$ \\
Straatsman et al. (1983) & - & $8.810^{-7} \exp \left[-\frac{24000}{\mathrm{R}}\left(\frac{1}{\mathrm{~T}}-\frac{1}{333}\right)\right]$ \\
Bravo et al. (1998) & - & $0.5910^{-6^{*}}$ \\
Palazzi and Converti (2001) & - & $\mathscr{D} / 6$ \\
This study & $0.4-0.8$ & $0.5 \mathscr{D}$ to $0.8 \mathscr{D}^{\text {t? }}$ \\
\hline
\end{tabular}

*Experimental values. Linko et al. (1981) - actinoplane cells immobilized in cellulose grains (conc. $\left.3.4 \mathrm{M} ; 60^{\circ} \mathrm{C}\right)$. Bravo et al. (1998) glucose isomerase immobilized in silica (conc. $2 \mathrm{M} ; 60^{\circ} \mathrm{C}$ ).

${ }^{*}$ calculated from Fig. 6.

Table 2: Molecular diffusivity of glucose in aqueous solution (Beenackers et al., 1986).

\begin{tabular}{|l|r|r|r|r|}
\hline $\boldsymbol{C}_{\boldsymbol{G}}\left(\mathbf{m o l} . \mathrm{m}^{-\mathbf{3}}\right)$ & 0 & 580 & 2620 & 4330 \\
\hline $\left.\mathscr{D} \mathbf{. 1 0} \mathbf{5}^{\mathbf{2}} \mathbf{c m}^{\mathbf{2}} \mathbf{- 1}\right)$ & 0.675 & 0.580 & 0.302 & 0.133 \\
\hline
\end{tabular}

\section{DESCRIPTION OF THE PROCESS - INTERMEDIATE CASE}

This second process is a decomposition reaction $(A \rightarrow B+C)$ where the products, species $B$ and $C$, are selectively adsorbed in the micropores of adsorbent particles. It is assumed that there is no diffusion of reagent species inside the particles, which have a bidisperse structure: porous microparticles grouped together by means of a binder such that macroporous pellets are generated. Figure 1 illustrates the length scales of this problem. Level 3 is the macroscale, on which there is a fluid phase, identified as the $\eta$-phase, around the porous particles, the $\omega$-region, packed into the reactor. Level 2 , which represents the intermediate scale, includes the macroporous region and involves a set of microparticles referred to as the $\sigma$-region. The $\sigma$ region is composed of micropores, as represented in level 1, and has a fluid phase $\gamma$ and an impermeable solid phase, the $\kappa$-phase. In this problem, the approach focuses on the mass transport taking place on the intermediate level. In this length scale, the

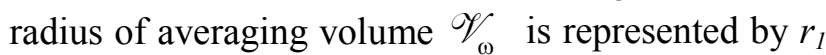
and the symbols $\ell_{\beta}$ and $\ell_{\sigma}$ are used to represent the characteristic length of the respective phases (Fig. 1).
The diffusion and adsorption problem under consideration can be described by the following differential equations and interfacial boundary conditions:

$$
\frac{\partial C_{i \beta}}{\partial t}=\nabla \cdot\left(\mathscr{D}_{i \beta} \nabla C_{i \beta}\right), \quad \text { in } \beta \text {-phase }
$$

B.C.1

$$
C_{i \beta}=C_{i \sigma}, \quad \text { in } \mathscr{A}_{\beta \sigma}
$$

B.C. 2

$$
\begin{aligned}
& -\mathbf{n}_{\sigma \beta} \cdot \mathscr{D}_{i \beta} \nabla C_{i \beta}=-\mathbf{n}_{\sigma \beta} \cdot \mathbf{D}_{i \sigma} \cdot \nabla C_{i \sigma}, \text { in } \delta \mathscr{A}_{\beta \sigma} \\
& \varepsilon_{\gamma}\left(1+\frac{\left.a_{v}\right|_{\gamma \kappa} K_{i}}{\varepsilon_{\gamma}}\right) \frac{\partial C_{i \sigma}}{\partial t}=\nabla \cdot\left(\mathbf{D}_{i \sigma} \cdot \nabla C_{i \sigma}\right),
\end{aligned}
$$

in $\sigma$-region

B.C.3

$$
C_{i \beta}=\mathscr{F}_{i}(\boldsymbol{r}, t), \quad \text { in } \mathscr{A}_{\beta e}
$$


B.C. 4

$C_{i \sigma}=\mathscr{E}_{i}(\boldsymbol{r}, t), \quad$ in $\mathscr{A}_{\sigma e}$

I.C.1

$C_{i \beta}=\mathscr{H} \mathscr{C}_{i}(\boldsymbol{r}), \quad \quad \quad t=0$

I.C.2

$C_{i \sigma}=\mathscr{O}_{i}(\boldsymbol{r}), \quad \quad t=0$

in which $\mathscr{A}_{\beta \sigma}$ is the interfacial area inside the microporous region and $\mathscr{A}_{\beta e}$ and $\mathscr{A}_{\sigma e}$ are the input and output areas associated with the averaging volume $\mathscr{T}_{\omega}$. The mass transport information from the microporous region is transferred to the macroporous scale through Eq.(19), in which $K_{i}$ is the adsorption equilibrium constant, assuming linear adsorption isotherms for the species. The parameters $\varepsilon_{\gamma}$ and $\mathbf{D}_{i \sigma}$ represent the porosity of a microparticle and the effective diffusivity tensor of species $i$ in the $\sigma$-region.

The volume-averaged transport equations for level 2 can be expressed as

$\varepsilon_{\beta} \frac{\partial\left\langle\mathrm{C}_{\mathrm{i} \beta}\right\rangle^{\beta}}{\partial \mathrm{t}}=$

$=\nabla \cdot\left[\mathscr{C}_{\beta}\left(\varepsilon_{\beta} \nabla\left\langle\mathrm{C}_{\mathrm{i} \beta}\right\rangle^{\beta}+\frac{1}{\mathscr{\mathscr { C }}} \int_{\mathrm{A}_{\beta \sigma}} \boldsymbol{n}_{\beta \sigma} \tilde{\mathrm{C}}_{\mathrm{i} \beta} \mathrm{dA}\right)\right]+$

$+\frac{1}{\mathscr{T}} \int_{A_{\beta \sigma}} \boldsymbol{n}_{\beta \sigma} \cdot \mathscr{O C}_{\beta} \nabla \mathrm{C}_{\mathrm{i} \beta} \mathrm{dA}$

$\varepsilon_{\sigma} \varepsilon_{\gamma}\left(1+\frac{\left.\mathrm{a}_{\mathrm{v}}\right|_{\gamma K} \mathrm{~K}_{\mathrm{i}}}{\varepsilon_{\gamma}}\right) \frac{\partial\left\langle\mathrm{C}_{\mathrm{i \sigma}}\right\rangle^{\sigma}}{\partial \mathrm{t}}=$

$=\nabla \cdot\left[\mathrm{D}_{\mathrm{i} \sigma \cdot} \cdot\left(\varepsilon_{\sigma} \nabla\left\langle\mathrm{C}_{\mathrm{i} \sigma}\right\rangle^{\sigma}+\frac{1}{\mathscr{\mathscr { T }}} \int_{\mathrm{A}_{\sigma \beta}} \boldsymbol{n}_{\sigma \beta} \tilde{\mathrm{C}}_{\mathrm{i} \sigma} \mathrm{dA}\right)\right]+$

$+\frac{1}{\mathscr{T}} \int_{\omega} \boldsymbol{n}_{\mathrm{A}_{\beta \sigma}} \cdot \mathrm{D}_{\mathrm{i} \sigma} \cdot \nabla \mathrm{C}_{\mathrm{i} \sigma} \mathrm{dA}$ in which the average values and volume fractions of the $\beta$-phase and $\sigma$-region are defined as shown in Eq.(6) and Eq.(7), respectively.

If the two average concentrations, $\left\langle C_{i \beta}\right\rangle^{\beta}$ and $\left\langle C_{i \sigma}\right\rangle^{\sigma}$, are sufficiently close to their equilibrium values, Eq.(24) and Eq.(25) can be combined and the interfacial flux terms eliminated. This mass transfer process can then be represented in terms of a oneequation model,

$$
\begin{aligned}
& \left(\varepsilon\left(1+\mathrm{K}_{\mathrm{i}}^{* *}\right)\right) \frac{\partial\left\{\mathrm{C}_{\mathrm{i}}\right\}}{\partial \mathrm{t}}= \\
& =\nabla \cdot\left[\mathscr{C}_{\beta}\left(\varepsilon_{\beta} \nabla\left\{\mathrm{C}_{\mathrm{i}}\right\}+\frac{1}{\mathscr{\mathscr { C }}} \int_{\mathrm{A}_{\beta \sigma}} n_{\beta \sigma} \tilde{\mathrm{C}}_{\mathrm{i} \beta} \mathrm{dA}\right]+\right. \\
& \left.+\mathrm{D}_{\mathrm{i} \sigma} \cdot\left(\varepsilon_{\sigma} \nabla\left\{\mathrm{C}_{\mathrm{i}}\right\}+\frac{1}{\mathscr{\mathscr { C }}} \int_{\mathrm{A}_{\beta \sigma}} \boldsymbol{n}_{\sigma \beta} \tilde{\mathrm{C}}_{\mathrm{i} \sigma} \mathrm{dA}\right)\right]
\end{aligned}
$$

in which the single concentration $\left\{C_{i}\right\}$ is referred to as the spatial averaged concentration, given by

$$
\left\{C_{i}\right\}=\frac{1}{\mathscr{Q}_{\omega}} \int_{\mathscr{\mathscr { V }}_{\omega}} C_{i} d V=\varepsilon_{\beta}\left\langle C_{i \beta}\right\rangle^{\beta}+\varepsilon_{\sigma}\left\langle C_{i \sigma}\right\rangle^{\sigma}
$$

In Eq.(26), we have made use of the nomenclature

$$
\varepsilon=\varepsilon_{\beta}+\varepsilon_{\sigma} \varepsilon_{\gamma} ; \quad K_{i}^{* *}=\frac{\left.\varepsilon_{\sigma} a_{v}\right|_{\gamma \kappa} K_{i}}{\varepsilon}
$$

Borges da Silva (2004) derived the time and length scale restrictions that must be satisfied if the one-equation model, and therefore the assumption of local mass equilibrium, is valid to describe this diffusion and adsorption process. These restrictions are related to the local mass equilibrium principle, which is discussed in Quintard and Whitaker (1998).

\section{Closure Problem of Intermediate Scale}

In order to obtain the closed form of Eq.(26), one needs to develop representations for the spatial deviation concentrations. Borges da Silva (2004) showed that $\widetilde{C}_{i \beta}$ and $\widetilde{C}_{i \sigma}$ can be expressed as 
$\widetilde{C}_{\beta}=\boldsymbol{b}_{\beta} \cdot \nabla\{C\} \quad ; \quad \widetilde{C}_{\sigma}=\boldsymbol{b}_{\sigma} \cdot \nabla\{C\}$

where $\boldsymbol{b}_{\beta}$ and $\boldsymbol{b}_{\sigma}$ are the closure variables of the intermediate scale. These variables can be determined by solving the boundary value problem,

$\nabla^{2} \boldsymbol{b}_{\beta}=0$

B.C.1

$\boldsymbol{b}_{\beta}=\boldsymbol{b}_{\sigma}, \quad$ in $\mathscr{A}_{\beta \sigma}$

B.C. 2

$-\boldsymbol{n}_{\sigma \beta} \cdot d \cdot \nabla b_{\sigma}=-\boldsymbol{n}_{\sigma \beta} \cdot \nabla b_{\beta}+\boldsymbol{n}_{\sigma \beta} \cdot(d-\mathbf{I})$,

in $\mathscr{e}_{\beta \sigma}$

$\nabla^{2} \boldsymbol{b}_{\sigma}=0$

Periodicity:

$\boldsymbol{b}_{\beta}\left(\boldsymbol{r}+\ell_{j}\right)=\boldsymbol{b}_{\beta}(\boldsymbol{r})$

$\boldsymbol{b}_{\sigma}\left(\boldsymbol{r}+\ell_{j}\right)=\boldsymbol{b}_{\sigma}(\boldsymbol{r}) \quad j=1,2,3$

where $d$ is a tensor given as

$d=\frac{\mathbf{D}_{i \sigma}}{\mathscr{D}_{i \beta}}$

The closure problem given by Eqs.(30)-(34) is assumed as quasi-steady. In addition, the concentration deviations are evaluated considering the local problem using a representative region of the intermediate scale and with a spatially periodic model of the porous medium such as that illustrated in Fig. 3. This procedure allows the use of the periodicity conditions shown in Eq.(34).

The representations given by Eq.(29) can be used to derive the closed form of Eq.(26), which can be expressed as

$$
\varepsilon\left(1+K_{i}^{* *}\right) \frac{\partial\{C\}}{\partial t}=\nabla \cdot\left(\mathbf{D}_{i}^{*} \cdot \nabla\{C\}\right)
$$

in which the effective diffusivity tensor of the intermediate scale is

$$
\begin{aligned}
& \mathrm{D}_{\mathrm{i}}^{*}=\left(\varepsilon_{\beta} \mathscr{D}_{\mathrm{i} \beta} \mathrm{I}+\varepsilon_{\sigma} \mathrm{D}_{\mathrm{i} \sigma}\right)+ \\
& +\frac{\left(\mathrm{D}_{\mathrm{i} \sigma}-\mathscr{D}_{\mathrm{i} \beta} \mathrm{I}\right)}{\mathscr{C}_{\omega}} \cdot \int_{\mathrm{A}_{\beta \sigma}} \boldsymbol{n}_{\sigma \beta} \boldsymbol{b}_{\sigma} \mathrm{dA}
\end{aligned}
$$

The diffusivity tensor of the intermediate scale under consideration can be predicted with the solution of the closure problem given by Eqs.(30)(34). The functional dependency of this tensor can be verified in Eq.(37), which includes contributions from (i) the molecular diffusivity of species in the $\beta$ phase (ii) the effective diffusivity tensor of species in the $\sigma$-region (iii) the void fractions of phases and (iv) the characteristics of the geometrical structure of the porous medium represented by the area integral of the term $\boldsymbol{n}_{\sigma \beta} . \boldsymbol{b}_{\sigma}$. Thus, it is necessary to solve the boundary value problem for the two vector fields, $\boldsymbol{b}_{\beta}$ and $\boldsymbol{b}_{\sigma}$.

This closure problem is solved by using the twodimensional square array of cylinders, as shown in Fig. 3a. This kind of arrangement provides an isotropic tensor, and therefore only a single component of the fields $\boldsymbol{b}_{\beta}$ and $\boldsymbol{b}_{o}$, such as $b_{\beta, x}$ and $b_{\sigma, x}$, respectively, needs to be evaluated to determine $\mathbf{D}_{i}^{*}$. The application of spatially periodic models facilitates the computational procedure and, in some cases, does not impose severe theoretical limitations (Ochoa-Tapia et al., 1986). However, the kind of periodic model chosen to solve the closure problem should be defined with some care, i.e., since the area integral of Eq.(37) functions as a filter, it is important to include the geometrical data from the medium to the representative region.

Some symmetry conditions can be imposed for the considered array and the computational domain is reduced to one quarter of the unit cell, as illustrated in Fig. 7. Figure 7 shows a scheme that exemplifies the computational mesh employed for solution of the closure variable fields and also presents the boundary conditions for $b_{\beta, x}$ and $b_{\sigma, x}$. Again, the differential equations are written in a general curvilinear coordinate system $(\eta-\xi$ system), allowing the numerical procedure to be carried out in a transformed plane (generalized coordinate system). 


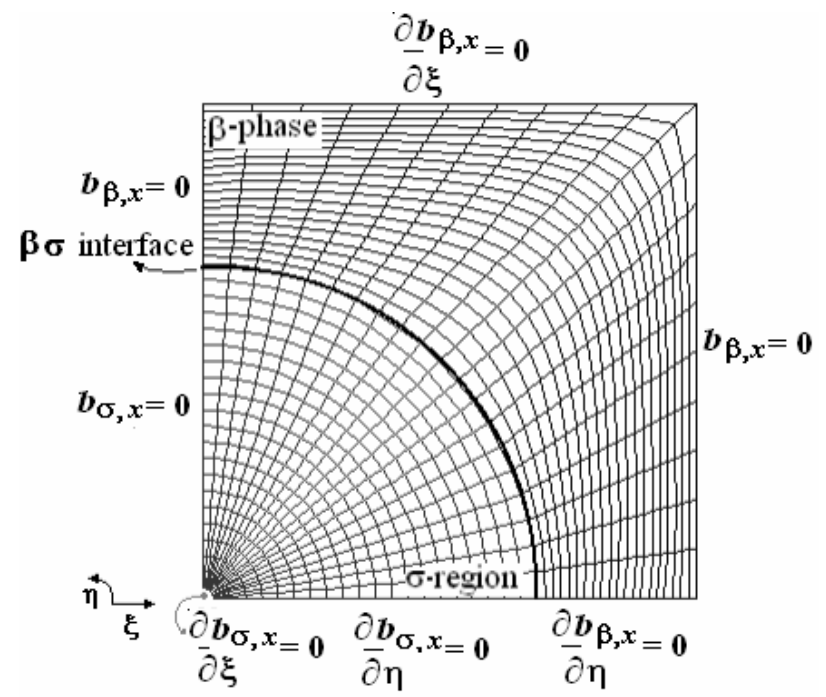

Figure 7: Mesh of the computational domain and boundary conditions for the solution of fields $b_{\beta, x}$ and $b_{\sigma, x}$. Generalized coordinates.

The boundary value problem given by Eqs. (30)(34) has been described with some generality to demonstrate equivalence with the case of the heat conduction problem in a biphasic system solved by Nozad et al. (1985). In the heat conduction problem, the tensor $d$ is specified as a scalar $(d)$ that corresponds to the ratio of the thermal conductivities $(k)$ of the two phases, the $\beta$-phase and the $\sigma$-region. Additionally, the periodic models in Nozad et al. represent the case of particles without contact, such as those illustrated in Fig. 3a, and the case of particles with contact, in which the $\sigma$-region has connections. The results obtained in this study are compared to the published results for the effective conductivity tensor, where $d$ assumes the value of the $k_{\delta} k_{\beta}$ ratio, and thus such a tensor is a scalar in this analysis.

The equation specifying the effective conductivity tensor in the biphasic process of Nozad et al. (1985) is given by

$$
\frac{\boldsymbol{K}_{e f}}{k_{\beta}}=\left(\varepsilon_{\beta}+\varepsilon_{\sigma} d\right) \boldsymbol{I}+\frac{(1-d)}{\mathscr{W}_{\omega}} \cdot \int_{A_{\beta \sigma}} \boldsymbol{n}_{\beta \sigma} \boldsymbol{b}_{\beta} d A
$$

and the effective diffusivity tensor for the intermediate scale of the studied system is given by

$$
\frac{\mathbf{D}_{i}^{*}}{\mathscr{D}_{i \beta}}=\left(\varepsilon_{\beta} \mathbf{I}+\varepsilon_{\sigma} d\right)+\frac{(\mathbf{I}-d)}{\mathscr{W}_{\omega}} \cdot \int_{A_{\beta \sigma}} \boldsymbol{n}_{\beta \sigma} \boldsymbol{b}_{\beta} d A
$$

Figure 8 shows the values for $\mathbf{K}_{e f} / k_{\beta}$ calculated in this study and the results published in the literature for several porosity values of the $\beta$-phase.
Nozad et al. (1985) demonstrated that, for calculation of the effective conductivity, the contact between the particles ( $\sigma$-region) in the porous medium is very important and results from unit cells for this array closely agree with the experimental results. In our case of adsorbent particles with a bidisperse structure, the solid porous medium is usually described as a set of porous microparticles, which can be assumed to have a spherical geometry. Thus, the spatially periodic model seems to be appropriate for the calculation of $\mathbf{D}_{i}^{*}$. It is worth mentioning that, for cases presented in the literature, three-dimensional arrays of spheres give slightly higher values than those for the analogous twodimensional versions (Whitaker, 1999).

In the cases studied, the finite volumes method is used to discretize the differential equations. The computational implementation is performed in FORTRAN language. The computational meshes with refinements equal to or above $40 \times 40$ (i.e., 1600 control volumes) are used in the simulations in such a way that no change is observed between different runs. The applied tolerance, which is defined as the ratio of the difference between the new value and the old value from the prior iteration to the new value of the variable from the latest iteration, is around $10^{-9}$. In Fig. 8, one can observe that the results obtained in this study are in good agreement with the results found in the literature, validating the numerical methodology. As commented upon in Whitaker (1999), the geometry slightly influences the effective conductivity $\mathbf{K}_{e f}$, which is dependent on the parameters $\varepsilon_{\beta}$ and $d$ in this medium, which is isotropic with respect to conduction. 


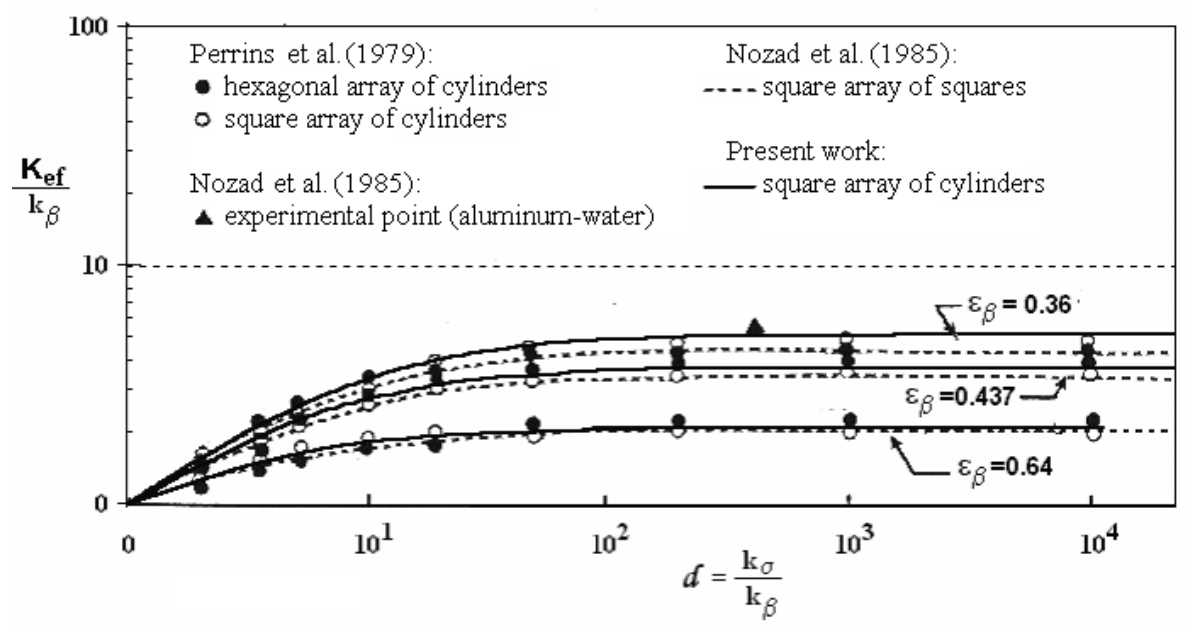

Figure 8: Effective thermal conductivity for the two-dimensional array of particles without contact.

For high values of $d$ (greater than 100), the numerical solution for this closure problem becomes complicated, because higher degrees of refinement for the computational meshes are required and therefore the computational time is prohibitive. In order to overcome this difficulty, the suggestion of Nozad et al. (1985) is followed, expressing the second boundary condition as

\section{B.C.2}

$-\boldsymbol{n}_{\sigma \beta} \cdot \nabla b_{\sigma, x}=-\lambda \boldsymbol{n}_{\sigma \beta} \cdot \nabla b_{\beta, x}+(1-\lambda) \boldsymbol{n}_{\sigma \beta} . \boldsymbol{i}$,

in $\mathscr{A \sigma}$

where $\lambda=d^{-1}$. The fields of the closure variables are given by

$b_{\beta, x}=b_{\beta, x}^{o}+\lambda b_{\beta, x}^{\prime}+\lambda^{2} b_{\beta, x}^{\prime \prime}+\ldots$

$b_{\sigma, x}=b_{\sigma, x}^{o}+\lambda b_{\sigma, x}^{\prime}+\lambda^{2} b_{\sigma, x}^{\prime \prime}+\ldots$

Once this numerical methodology has been corroborated, the closure problem of the intermediate scale is solved to provide the theoretical prediction of effective diffusivity in the $\beta-\sigma$ system. The numerical solutions of the fields $b_{\beta}$ and $b_{\sigma}$ for the two-dimensional model of cylinders in a squared arrangement are used in Eq.(39). Figure 9 shows the numerical results of $\boldsymbol{D}_{\mathbf{i}}^{*}$ obtained from the closure problem associated with the method of volume averaging applied in the mass transfer problem on the intermediate scale. One can verify the ratio of isotropic effective diffusivity (where $\mathbf{D}_{i}^{*}=\boldsymbol{D}_{i}^{*} \mathbf{I}$ ) to diffusivity $\mathscr{D}_{i \beta}$ as a function of parameter $d$, the ratio of isotropic effective diffusivity in the $\sigma$-phase (where $\mathbf{D}_{\mathbf{i} \sigma}=\boldsymbol{D}_{\mathbf{i} \sigma} \mathbf{I}$ i) to diffusivity in the $\beta$-phase, and of the volumetric fraction of the continuous $\beta$ phase.

Since the diffusivity of species in the $\beta$-phase can be specified as the molecular diffusivity of species, and therefore must be greater than the effective diffusivity tensor in the $\sigma$-region, the values for parameter $d$ are less than unity. In Fig. 9, one can observe that for lower values of the volumetric fraction of the $\beta$-phase, the effective diffusivity tensor of the intermediate scale decreases. This probably occurs due to difficulties related to the diffusion of the species in the geometrical structure of the porous medium. When parameter $d$ tends to unity, the effect of the volume fraction $\varepsilon_{\beta}$ is not significant, and as expected, the value of $\boldsymbol{D}_{\mathbf{i}}^{*}$ tends to the molecular diffusivity of the species. Experimental data for this system are not presented in this paper. Ochoa-Tapia et al. (1986) and Wood and Whitaker (2000) derived the mathematical formulation of diffusion and reaction in biofilms and cellular media and solved closure problems similar to those presented herein when the mass transfer resistance in the cellular wall was negligible. For such a case, good agreement between the experimental results of the effective diffusivity in cellular media, the biphasic system, and the theoretical values obtained from the solution of the closure variable fields has been verified. Details can be found in Wood and Whitaker (2000). 


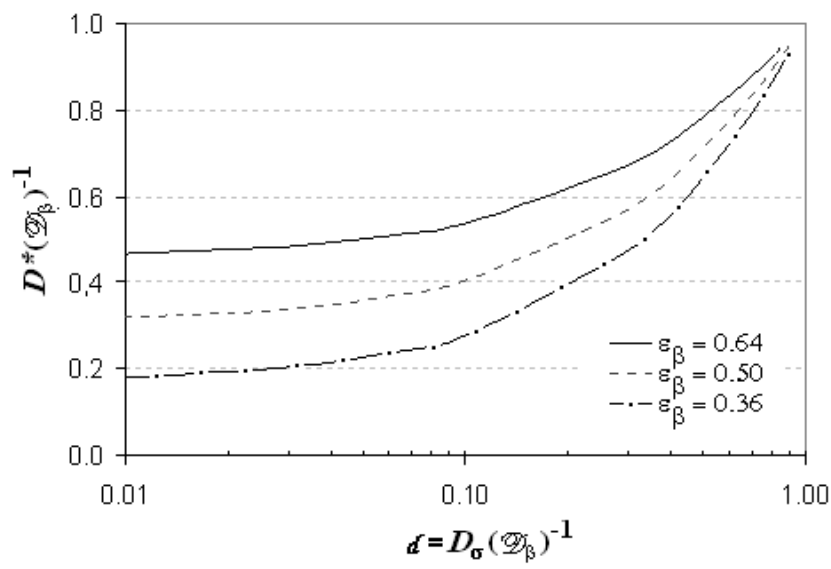

Figure 9: Effective diffusivity in the $\beta-\sigma$ system as a function of the ratio of diffusivities of the phases for different values of the volume fraction of the $\beta$-phase.

This study reveals the possibility of obtaining theoretically the effective diffusivity tensor of the intermediate scale of the $\beta-\sigma$ system, i.e., predicting the diffusivity inside particles with a bidisperse structure. It is worth mentioning that this effective transport coefficient is deduced for the one-equation model, and therefore the hypothesis of local mass equilibrium is considered valid. As a consequence, such a situation can be seen as a fusion of the $\beta$ phase and the $\sigma$-region to make up a single homogeneous medium on this scale. This approach leads to the obtention of a direct equivalence between the effective coefficient of a homogeneous particle and the effective coefficient calculated in this study.

\section{CONCLUSIONS}

In this paper, mass transport equations were presented in the form of volume-averaged mass conservation equations for two processes: (i) the governing transport equation for the porous region, the microscale, of the catalyst in a reactive process and (ii) the governing transport equation for the macroporous region, the intermediate length scale, of a bidisperse particle in an adsorptive process. The mathematical formulation in both situations studied was performed using the method of volume averaging. This procedure provided the transport equation of the reactive/adsorptive multiphase flow using information from the microscopic to the macroscopic scale

The application of the method of volume averaging enabled derivation of the closure problems. Two closure problems could be solved using the finite volumes methodology and spatially periodic models of porous media. The numerical solution of these problems allowed determination of closure variables, which were used to predict the effective diffusivity tensors for the $\gamma$ - $\kappa$ system in the porous catalyst and the $\beta-\sigma$ system in the adsorbent with the bidisperse structure. It was shown that the solutions are dependent on the geometrical structure of the porous medium, and even if simple arrays of spatially periodic models are used to solve closure problems, coherent estimates of the effective transport coefficients can be obtained. The volume averaging procedure was shown to be useful for application of multiphase flow in porous media, since it is possible to compare the theoretical results and the experimental data without any adjustable parameters.

\section{ACKNOWLEDGEMENTS}

Financial support from the Conselho Nacional de Desenvolvimento Científico e Tecnológico (CNPq) and the Coordenação de Aperfeiçoamento de Pessoal de Nível Superior (CAPES) is gratefully acknowledged.

\section{NOMENCLATURE}

$\left.a_{v}\right|_{\gamma \kappa} \quad \gamma-\kappa$ interfacial area per unit $\quad \mathrm{m}^{-1}$ volume $\mathscr{T}_{\sigma}$ or $\mathscr{W}_{\omega}$,

$\mathscr{A}_{\gamma \kappa}$ area of the $\gamma$ - $\mathrm{k}$ interface $\mathrm{m}^{2}$ contained within volume $\mathscr{T}_{\sigma}$ or $\mathscr{W}_{\omega}$,

$\mathcal{A}_{\gamma e}$ area of the entrances and exits $\mathrm{m}^{2}$ for the $\gamma$ phase, 
$\mathscr{A}_{\beta \sigma} \quad$ area of the $\beta-\sigma$ interface contained within volume $\mathscr{Q}_{\omega}$,

$\mathcal{A}_{\beta e} \quad$ area of the entrances and exits for the $\beta$ phase,

$\mathcal{A}_{\sigma e}$ area of the entrances and exits
for the $\sigma$ region,

$\mathcal{A}_{\sigma e}$ area of the entrances and exits
for the $\sigma$ region,

b closure variable,

$\boldsymbol{b}_{x} \quad$ component of the closure variable in the $x$ direction,

$C_{i x} \quad$ punctual concentration of species $i$ in the $x$ phase or region,

$\left\langle C_{i x}\right\rangle^{x}$ intrinsic average concentration of species $i$ in the $x$ phase or region,

$\widetilde{C}_{i x} \quad$ spatial deviation concentration of species $i$ in the $x$ phase or region, spatial averaged concentration in $\left\{C_{i}\right\}$ the local mass equilibrium condition of species $i$,

$d \quad$ ratio of the thermal conductivities

$\boldsymbol{d}$ ratio of the effective diffusivity and molecular diffusivity

$\mathscr{D}_{x}$ molecular diffusivity of species $i$ in the $x$ phase or region,

$\mathbf{D}_{i \sigma} \quad$ effective diffusivity tensor of species $i$ in the $\sigma$ region,

$\left.\boldsymbol{D}_{\text {ef } i}\right|_{\gamma \kappa}$ effective diffusivity tensor on

$\boldsymbol{D}_{i}{ }^{*} \quad$ the microscale of species $i$, effective diffusivity tensor on the intermediate scale of species $i$,

\section{I unit tensor}

$k_{x} \quad$ thermal conductivity in the $x$ phase or region,

$\mathbf{K}_{\text {ef }}$ effective thermal conductivity tensor,

$\ell_{j} \quad$ lattice vectors $(j=1,2,3)$,

$\ell_{x} \quad$ characteristic length associated with the $x$ phase or region,

$L \quad$ characteristic length,

$\boldsymbol{n}_{\mathbf{y x}} \quad$ outwardly directed unit normal vector pointing from the $y$ phase (or region) toward the $x$ phase (or region)

$r \quad$ position vector,

$r_{o} \quad$ radius of the averaging volume on the microscale,

$r_{1}$ radius of the averaging volume on the intermediate scale,

\begin{tabular}{|c|c|c|c|}
\hline $\mathrm{m}^{2}$ & $R_{i}$ & reaction rate of species $i$, & $\mathrm{kmol} / \mathrm{m}^{2} \mathrm{~s}$ \\
\hline $\mathrm{m}^{2}$ & $s_{i x}$ & closure variable associated with & $\mathrm{s} / \mathrm{m}$ \\
\hline $\mathrm{m}^{2}$ & $t$ & $\begin{array}{l}\text { the } x \text { region or phase, species } i \text {, } \\
\text { time, }\end{array}$ & \\
\hline & $\begin{array}{l}V \\
V\end{array}$ & $\begin{array}{l}\text { volume, } \\
\text { volume of the } x \text { phase or region }\end{array}$ & $\mathrm{m}^{3}$ \\
\hline $\mathrm{m}$ & & contained within the averaging & \\
\hline $\mathrm{m}$ & $\mathscr{W}$ & $\begin{array}{l}\text { volume, } \\
\text { averaging volume, }\end{array}$ & $\mathrm{m}^{3}$ \\
\hline $\mathrm{kmol} / \mathrm{m}^{3}$ & $x^{\sigma}$ & position vector locating the & $\mathrm{m}$ \\
\hline $\mathrm{kmol} / \mathrm{m}^{3}$ & & $\begin{array}{l}\text { centroid of the averaging } \\
\text { volume, }\end{array}$ & \\
\hline $\mathrm{kmol} / \mathrm{m}^{3}$ & $\mathbf{y}_{x}$ & $\begin{array}{l}\text { position vector locating points } \\
\text { relative to the centroid in the } x \\
\text { phase or region, }\end{array}$ & $\mathrm{m}$ \\
\hline
\end{tabular}

$\mathrm{kmol} / \mathrm{m}^{3} \quad$ Greek Letters

\begin{tabular}{|c|c|c|}
\hline & $\beta$ & intermediate scale phase \\
\hline$(-)$ & $\varepsilon_{x}$ & $\begin{array}{l}\text { volume fraction of the } x \text { phase } \\
\text { or region }\end{array}$ \\
\hline & $\gamma, \kappa$ & microscale phases \\
\hline & $\eta$ & macroscale phase \\
\hline $\mathrm{m}^{2} / \mathrm{s}$ & $\sigma$ & intermediate scale region \\
\hline & $\omega$ & macroscale region \\
\hline $\mathrm{m}^{2} / \mathrm{s}$ & $\psi_{x}$ & $\begin{array}{l}\text { closure variable associated } \\
\text { with the } x \text { phase or region, } \\
\mathrm{kmol} / \mathrm{m}^{3}\end{array}$ \\
\hline
\end{tabular}

$\mathrm{kg} \mathrm{m} / \mathrm{s}^{3} \mathrm{~K}$

$(-)$

$\mathrm{kg} \mathrm{m} / \mathrm{s}^{3} \mathrm{~K}$

$\mathrm{m}$

$\mathrm{m}$

$\mathrm{m}$

$\mathrm{m}$

$\mathrm{m}$

$\mathrm{m}$

\section{REFERENCES}

Asif, M. and Abasaeed, A. E., Modeling of Glucose Isomerization in a Fluidized Bed Immobilized Enzyme Bioreactor, Bioresource Technology, 64, p. 229-235 (1998).

Beenackers, J. A. W. M., Kuster, B. F. M., and van der Baan, H. S., Adsorption of Carbohydrates on Anion Exchangers, Applied Catalysis, 23, p. 183197 (1986).

Borges da Silva, E. A., Estudo da Transferência de Massa em Unidades de Leito Móvel Simulado Reativo. Ph.D. diss., Federal University of Santa Catarina (2004).

Bravo, V., Jurado, E., Luzón, G., and Cruz, N., Kinetics of Fructose-Glucose Isomerization with Sweetzyme Type A, The Can. Jour. of Chem. Eng., 76, p. 778-783 (1998).

Brenner, H., Dispersion Resulting from Flow through Spatially Periodic Porous Media, Trans. Roy. Soc. (London), 297, p. 81-133 (1980). 
Chang, H. C., Effective Diffusion and Conduction in Two Phase Media: A Unified Approach, A.I.Ch.E. Journal, 29, p. 846-853 (1983).

Crapiste, G. H., Rotstein, E., and Whitaker, S., A General Closure Scheme for the Method of Volume Averaging, Chem. Eng. Sci., 41, No. 2, p. 227-235 (1986).

Guelli U. de Souza, S. M. A. and Whitaker, S., Mass Transfer in Porous Media with Heterogeneous Chemical Reaction, Brazilian Journal of Chemical Engineering, 20, No.2, p. 191-199 (2003).

Linko, P., Poutanen, K., and Linko, Y., Reactor Performance in Glucose Isomerization by Cellulose Bead Immobilized Actinoplanes Cells, Journal of Molecular Catalysis, 13, p. 263-278 (1981).

Maliska, C. R., Transferência de Calor e Mecânica dos Fluidos Computacional. LTC - Livros Técnicos e Científicos S.A., Rio de Janeiro, RJ (1995).

Nguyen, V. V., Gray, W. G., Pinder, G. F., Botha, J. F., and Crerar, D. A., A Theoretical Investigation on the Transport of Chemicals in Reactive Porous Media, Water Resources Research, 18, No. 4, p. 1149-1156 (1982).

Nozad, I., Carbonell, R. G., and Whitaker, S., Heat Conduction in Multiphase Systems I. Theory and Experiment for Two-phase Systems, Chem. Eng. Sci., 40, p. 843-855 (1985).

Ochoa-Tapia, J. A., Stroeve, P., and Whitaker, S., Diffusion and Reaction in Cellular Media, Chem. Eng. Sci., 41, No. 12, p. 2999-3013 (1986).

Ochoa-Tapia, J. A., Stroeve, P., and Whitaker, S., Diffusive Transport in Two-phase Media: Spatially Periodic Models and Maxwell's Theory for Isotropic and Anisotropic Systems, Chem. Eng. Sci., 49, No. 5, p. 709-726 (1994).

Palazzi, E. and Converti, A., Evaluation of Diffusional Resistances in the Process of Glucose Isomerization to Fructose by Immobilized Glucose Isomerase, Enzyme and Microbial Technology, 28, p. 246-252 (2001).

Perrins, W. T., McKenzie, D. R., and McPhedran, R. C., Transport-Properties of Regular Arrays of Cylinders. Proc. Roy. Soc. Lond. A369, p. 207225 (1979).
Plumb, O. A. and Whitaker, S., Diffusion, Adsorption, and Dispersion in Heterogeneous Porous Media: The Method of Large-Scale Averaging. In Dynamics of Fluids in Hierarchical Porous Media, J. H. Cushman, Academic Press, New York (1990).

Quintard, M. and Whitaker, S., Transport in Ordered and Disordered Porous Media: Volume-Averaged Equations, Closure Problems and Comparison with Experiments, Chem. Eng. Sci., 48, No. 14, p. 2537-2564 (1993).

Quintard, M. and Whitaker, S., Convection, Dispersion and Interfacial Transport of Contaminants: Homogeneous Porous Media, Advances in Water Resources, 17, p. 221-239 (1994).

Quintard, M. and Whitaker, S., Transport in Chemically and Mechanically Heterogeneous Porous Media. IV: Large-Scale Mass Equilibrium for Solute Transport with Adsorption, Adv. in Water Research, 22, No. 1, p. 33-57 (1998).

Ryan, D., Carbonell, R. G., and Whitaker, S., A Theory of Diffusion and Reaction in Porous Media, A.I.Ch.E. Symposium Series, 77, No. 202, p. 46-62 (1981).

Straatsma, J., Vellenga, K., Wilt, H. J., and Joosten, G. H., Isomerization of Glucose to Fructose. 2. Optimization of Reaction Conditions in the Production of High Fructose Syrup by Isomerization of Glucose Catalyzed by a Whole Cell Immobilized Glucose Isomerase Catalyst, Ind. Eng. Chem. Process Des. Dev., 22, 356-361 (1983).

Ulson de Souza, A. A. and Whitaker, S., The Modeling of a Textile Dying Process Utilizing the Method of Volume Averaging, Brazilian Journal of Chemical Engineering, 20, No.4, p. 445-453 (2003).

Whitaker, S., Theory and Applications of Transport in Porous Media: The Method of Volume Averaging. London: Kluwer Academic (1999).

Wood, B. D. and Whitaker, S., Diffusion and Reaction in Biofilms, Chem. Eng. Sci., 53, No. 3, p. 397-425 (1998).

Wood, B. D. and Whitaker, S., Multi-species Diffusion and Reaction in Biofilms and Cellular Media, Chem. Eng. Sci., 55, p. 3397-3418 (2000). 